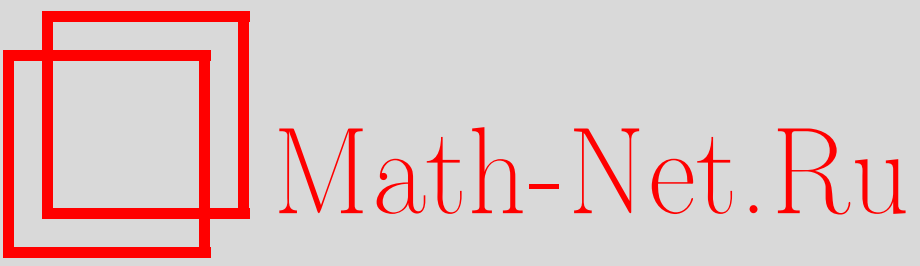

Л. А. Игнаточкина, Ю. А. Горгинян, Примеры аффинно-метрических структур на почти эрмитовом многообразии, Итоги науки и техн. Сер. Соврем. мат. и ее прил. Темат. обз., 2020, том 181, 30-40

DOI: https://doi.org/10.36535/0233-6723-2020-181-30-40

Использование Общероссийского математического портала Math-Net.Ru подразумевает, что вы прочитали и согласны с пользовательским соглашением

http: //www. mathnet.ru/rus/agreement

Параметры загрузки:

IP : 35.173 .219 .149

26 апреля 2023 г., 10:00:20 


\title{
ПРИМЕРЫ АФФИННО-МЕТРИЧЕСКИХ СТРУКТУР НА ПОЧТИ ЭРМИТОВОМ МНОГООБРАЗИИ
}

\author{
(c) 2020 г. Л. А. ИГНАТОЧКИНА, Ю. А. ГОРГИНЯН
}

\begin{abstract}
АннотАция. На почти эрмитовом многообразии при помощи ковариантного дифференциала почти комплексной структуры в римановой связности псевдоримановой метрики построено аффинное подпространство аффинных связностей. Найдены возможные размерности этого пространства. Для 8-мерного пространства найдены многомерные плоскости связностей, задающих постримановы геометрии. Найдены связности, для которых тензор кручения определяется только структурным, либо только виртуальным тензором. Найдены связности, в которых ковариантный дифференциал почти комплексной структуры определяется только структурным, либо только виртуальным тензором.
\end{abstract}

Ключевые слова: аффинная связность, почти эрмитово многообразие, конформное преобразование.

\section{EXAMPLES OF AFFINE-METRIC STRUCTURES ON AN ALMOST HERMITIAN MANIFOLD}

\author{
(c) 2020 L. A. IGNATOCHKINA, YU. A. GORGINYAN
}

\begin{abstract}
On an almost Hermitian manifold, we construct an affine subspace of affine connections by using the covariant differential of the almost complex structure on the Riemannian connection of a pseudo-Riemannian metric. We find possible dimensions of this space. For the 8-dimensional space, we find multidimensional planes of connections that determine post-Riemannian geometries. We also find connections for which the torsion tensor is determined only by the structural tensor or only by the virtual tensor. We fond connections in which the covariant differential of the almost complex structure is determined only by the structural tensor or only by the virtual tensor.
\end{abstract}

Keywords and phrases: affine connection, almost Hermitian manifold, conformal transformation.

AMS Subject Classification: 53B05, 51E15

1. Введение. Аффинно-метрические структуры, т.е. пары, состоящие из псевдоримановой метрики и произвольной аффинной связности, изучаются в современной дифференциальной геометрии и находят применение в области теоретической физики (см., например, $[1,6])$. Особый интерес представляют связности, тензор неметричности которых представим в виде тензорного произведения 1-формы (формы Вейля) и псевдоримановой метрики. Говорят, что такие связности (вместе с метрикой) задают геометрию Римана-Картана-Вейля. В зависимости от обращения в нуль тензора неметричности и тензора кручения связности среди геометрий Римана-Картана-Вейля выделяются геометрии Римана-Картана и геометрии Римана-Вейля. Все три вида геометрии называются постримановыми геометриями.

Другой структурой, задаваемой на гладком многообразии, является почти эрмитова структура, т.е. пара, состоящая из почти комплексной структуры $J$ и согласованной с ней псевдоримановой метрики. Ковариантный дифференциал $J$ является тензорным полем типа $(2,1)$. Используя его 
в качестве тензора аффинной деформации, можно построить аффинное подпространство аффинных связностей. Эти связности вместе с псевдоримановой метрикой почти эрмитовой структуры задают аффинно-метрическую структуру на многообразии. Тогда, во-первых, встает вопрос о свойствах тензора кручения и тензора неметричности построенных связностей. В частности, встает вопрос о том, какие постримановы геометрии задают построенные связности.

Для почти эрмитовой структуры определяются два тензорных поля: структурный и виртуальный тензоры. С их помощью проводится классификация почти эрмитовых структур. Поэтому, во-вторых, встает вопрос о существовании связностей, тензор кривизны которых определяется либо только структурным, либо только виртуальным тензором. В-третьих, аналогичный вопрос встает для ковариантного дифференциала $J$ в построенных связностях. Так как структурный тензор инвариантен при конформных преобразованиях метрики, то в случае, когда тензор кручения и ковариантный дифференциал $J$ определяются только структурным тензором, они также будут конформно инвариантными.

2. Почти эрмитовы многообразия. Напомним (см. [3]), что почти эрмитовым многообразием называется гладкое многообразие $M$, на котором фиксированы тензорные поля $(J, g)$, где $J$ - тензорное поле типа $(1,1), J^{2}=-\mathrm{id}, g$ - псевдориманова метрика на $M$, для которой выполняется соотношение $g(J X, J Y)=g(X, Y), X, Y$-векторные поля на $M$. Тензорное поле $J$ называется почти комплексной структурой. Обозначим размерность $M$ через $2 n, n>1$.

На почти эрмитовом многообразии определяется (см. [3,5]) кэлерова форма $F(X, Y)=g(J X, Y)$ и форма Ли $\alpha(X)=1 /(1-n) \delta F(J X)$, где $\delta$-оператор кодифференцирования. Векторное поле $\xi$, для которого $g(\xi, X)=\alpha(X)$, называется вектором Ли.

Пусть индексы $a, b, c, d, \ldots$ принимают значения от 1 до $n$, индексы $i, j, k, l, \ldots$ принимают значения от 1 до $2 n$ и $\hat{a}=a+n$.

Напомним (см. [3]), что для почти эрмитова многообразия строятся так называемые $A$-реперы $p=\left(m, \varepsilon_{1}, \ldots, \varepsilon_{n}, \varepsilon_{\hat{1}}, \ldots, \varepsilon_{\hat{n}}\right)$ (короче, $\left.p=\left(m, \varepsilon_{a}, \varepsilon_{\hat{a}}\right)\right)$ следующим образом. Рассмотрим репер $\left(m, e_{1}, \ldots, e_{n}\right)$, ортонормированный относительно эрмитовой формы

$$
\langle\langle X, Y\rangle=g(X, Y)+i g(X, J Y),
$$

$X, Y \in T_{m}(M)$, где $T_{m}(M)$ рассматривается как комплексное векторное пространство с операцией умножения на комплексное число по формуле $i X=J X$. Тогда векторы $A$-репера получаются по формулам $\varepsilon_{a}=\sqrt{2} \sigma\left(e_{a}\right), \varepsilon_{\hat{a}}=\sqrt{2} \bar{\sigma}\left(e_{a}\right)$, где

$$
\sigma=\frac{1}{2}(\mathrm{id}-i J), \quad \bar{\sigma}=\frac{1}{2}(\mathrm{id}+i J) .
$$

Задание тензорного поля на многообразии $M$ равносильно заданию системы функций на пространстве расслоения реперов. Эти функции называются компонентами тензорного поля на пространстве расслоения $A$-реперов. В частности, для почти комплексной структуры $J$ система функций $\left\{J_{j}^{i}(p)\right\}$ на пространстве расслоения всех комплексных реперов определяется формулой $J_{m}^{\mathbb{C}}\left(\varepsilon_{i}\right)=J_{i}^{j}(p) \varepsilon_{j}$, а для псевдоримановой метрики $g$ система функций $\left\{g_{i j}(p)\right\}$ определяется формулой $g_{i j}(p)=g_{m}^{\mathbb{C}}\left(\varepsilon_{i}, \varepsilon_{j}\right)$. Здесь $p=\left(m, \varepsilon_{i}\right), m \in M$ - произвольный комплексный репер, знак $\mathbb{C}$ обозначает комплексификацию тензора.

На пространстве расслоения $A$-реперов эти системы функций имеют простой вид:

$$
\left(J_{j}^{i}\right)=\left(\begin{array}{cc}
i I_{n} & 0 \\
0 & -i I_{n}
\end{array}\right) \quad\left(g_{i j}\right)=\left(\begin{array}{cc}
0 & I_{n, s} \\
I_{n, s} & 0
\end{array}\right)
$$

где $I_{n}$ - единичная матрица, а $I_{n, s}$ - матрица порядка $n$, на диагонали стоят числа 1 в количестве $n-s$ штук и -1 в количестве $s$ штук, $2 s$ - индекс метрики $g$ (см. [3]).

Обозначим через $\left\{\alpha_{a}, \alpha_{\hat{a}}\right\}$ компоненты формы Ли на пространстве расслоения $A$-реперов, а через $\left\{\xi^{b}, \xi^{\hat{b}}\right\}$ - компоненты вектора Ли $\xi$ на пространстве расслоения $A$-реперов. В силу (2) получаем

где $\varepsilon(a)=g_{a \hat{a}}$.

$$
\xi^{a}=\varepsilon(a) \alpha_{\hat{a}}, \quad \xi^{\hat{a}}=\varepsilon(a) \alpha_{a},
$$


Для почти эрмитова многообразия определяются тензорные поля типа $(2,1)$ по формулам

$$
B(X, Y)=\frac{1}{4}\left(\nabla_{Y}(J)(J X)-\nabla_{J Y}(J) X\right), \quad C(X, Y)=-\frac{1}{4}\left(\nabla_{J Y}(J) X+\nabla_{Y}(J)(J X)\right),
$$

где $\nabla$ - риманова связность псевдоримановой метрики $g$. Они называются виртуальным тензором и структурным тензором соответственно. Их ненулевые компоненты на пространстве расслоения $A$-реперов связаны с компонентами $\left\{J_{j, k}^{i}\right\}$ ковариантного дифференциала $J$ в римановой связности по формулам (см. [3])

$$
\begin{aligned}
& B^{a b}{ }_{c}=-\frac{i}{2} \varepsilon(b) J_{\hat{b}, c}^{a}, \quad B_{a b}{ }^{c}=\frac{i}{2} \varepsilon(b) J_{b, \hat{c}}^{\hat{a}}, \quad \overline{B_{a b}{ }^{c}}=B^{a b}{ }_{c} . \\
& C_{a b c}=-\frac{i}{2} \varepsilon(b, c) J_{b, c}^{\hat{a}}, \quad C^{a b c}=\frac{i}{2} \varepsilon(b, c) J_{\hat{b}, \hat{c}}^{a}, \quad \overline{C_{a b c}}=C^{a b c},
\end{aligned}
$$

где $\varepsilon(b, c)=g_{b \hat{b}} g_{c \hat{c}}$. Кроме того, остальные компоненты ковариантного дифференциала $J$ равны нулю:

$$
J_{b, k}^{a}=J_{\hat{b}, k}^{\hat{a}}=0 .
$$

Компоненты вектора Ли и виртуального тензора связаны равенствами (см. [3])

$$
\xi^{a}=\frac{2}{n-1} B_{b}^{a b}, \quad \xi^{\hat{a}}=\frac{2}{n-1} B_{a b}{ }^{b} .
$$

Для классификации используется также альтернированный структурный тензор, который также называют структурным тензором. Его компоненты имеют вид

$$
B_{a b c}=C_{a[b c]}, \quad B^{a b c}=C^{a[b c]} .
$$

Отметим, что в силу кососимметричности компонент $C_{a b c}$ по первым двум индексам равенство $C_{a b c}=0$ равносильно равенству $B_{a b c}=0$. Кроме того, из (8) непосредственно следует, что

$$
C_{a b c}=B_{a b c}+B_{b c a}+B_{c b a} .
$$

Симметризуя это тождество по индексам $b, c$ и учитывая, что $B_{a b c}$ кососимметричны по последним двум индексам, получим

$$
C_{a(b c)}=2 B_{(b c) a} .
$$

Замечание 1. Во введенных обозначениях на пространстве расслоения $A$-реперов

$$
\varepsilon(c) J_{b, a}^{\hat{c}}+\varepsilon(b) J_{c, a}^{\hat{b}}=0, \quad B^{c b}{ }_{a}+B^{b c}{ }_{a}=0 .
$$

Доказательство. Из условия согласованности $g(J X, J Y)=g(X, Y)$ метрики $g$ и почти комплексной структуры $J$ имеем $g(J Y, Z)+g(Y, J Z)=0$. Применим к этому тождеству оператор ковариантного дифференцирования $\nabla_{X}$ :

$$
g\left(\nabla_{X}(J) Y, Z\right)+g\left(Y, \nabla_{X}(J) Z\right)=0 .
$$

На пространстве расслоения $A$-реперов получим

$$
g_{l k} J_{j, i}^{l}+g_{j l} J_{k, i}^{l}=0 .
$$

В частности, для $i=a, j=b, k=c$ с учетом (2) имеем

$$
\varepsilon(c) J_{b, a}^{\hat{c}}+\varepsilon(b) J_{c, a}^{\hat{b}}=0 .
$$

Для $i=a, j=\hat{b}, k=\hat{c}$ с учетом (2) и (5) имеем

$$
\varepsilon(c) J_{\hat{b}, c}^{c}+\varepsilon(b) J_{\hat{c}, a}^{b}=0,
$$

т.е.

$$
\varepsilon(c) \varepsilon(b) B^{c b}{ }_{a}+\varepsilon(b) \varepsilon(c) B^{b c}{ }_{a}=0 .
$$

Сокращая на $\varepsilon(c) \varepsilon(b)$, получаем требуемое равенство. 
3. Аффинное подпространство аффинных связностей почти эрмитова многообразия. Пусть дано почти эрмитово многообразие. Обозначим через $\nabla$ риманову связность псевдоримановой метрики $g$. Рассмотрим множество связностей $\Delta$

$$
\begin{aligned}
\Delta_{X} Y=\nabla_{X} Y+\mu_{1} \nabla_{X}(J) Y+\mu_{2} \nabla_{Y}(J) X+\mu_{3} \nabla_{J X}(J) Y+\mu_{4} \nabla_{Y}(J)(J X)+ \\
\quad+\mu_{5} \nabla_{X}(J)(J Y)+\mu_{6} \nabla_{J Y}(J) X+\mu_{7} \nabla_{J X}(J)(J Y)+\mu_{8} \nabla_{J Y}(J)(J X) .
\end{aligned}
$$

Это множество будет аффинным подпространством в аффинном пространстве всех аффинных связностей. Выясним, какая у него размерность.

Составим линейную комбинацию

$$
\begin{aligned}
\mu_{1} \nabla_{X}(J) Y+\mu_{2} \nabla_{Y}(J) X+\mu_{3} \nabla_{J X}(J) Y & +\mu_{4} \nabla_{Y}(J)(J X)+\mu_{5} \nabla_{X}(J)(J Y)+ \\
& +\mu_{6} \nabla_{J Y}(J) X+\mu_{7} \nabla_{J X}(J)(J Y)+\mu_{8} \nabla_{J Y}(J)(J X)=0 .
\end{aligned}
$$

На пространстве расслоения $A$-реперов получим (здесь $\left\{J_{i, j}^{k}\right\}$ - компоненты $\nabla J$ на пространстве расслоения $A$-реперов)

$$
\mu_{1} J_{j, i}^{k}+\mu_{2} J_{i, j}^{k}+\mu_{3} J_{j, l}^{k} J_{i}^{l}+\mu_{4} J_{l, j}^{k} J_{i}^{l}+\mu_{5} J_{l, i}^{k} J_{j}^{l}+\mu_{6} J_{i, l}^{k} J_{j}^{l}+\mu_{7} J_{l, t}^{k} J_{j}^{l} J_{i}^{t}+\mu_{8} J_{l, t}^{k} J_{i}^{l} J_{j}^{t}=0 .
$$

В частности, если $i=a, j=b, k=c$, то из (12) получаем тождество $0=0$. Если $i=\hat{a}, j=b$, $k=c$, то получаем

$$
\left(\mu_{2}+\mu_{8}+i\left(\mu_{6}-\mu_{4}\right)\right) J_{\hat{a}, b}^{c}=0 .
$$

Если $i=a, j=\hat{b}, k=c$, то из (12) получаем

$$
\left(\mu_{1}+\mu_{7}+i\left(\mu_{3}-\mu_{5}\right)\right) J_{\hat{b}, a}^{c}=0 .
$$

Если $i=a, j=b, k=\hat{c}$, то из (12) получаем

$$
J_{b, a}^{\hat{c}}\left(\mu_{1}-\mu_{7}+i\left(\mu_{3}+\mu_{5}\right)\right)+J_{a, b}^{\hat{c}}\left(\mu_{2}-\mu_{8}+i\left(\mu_{4}+\mu_{6}\right)\right)=0 .
$$

Остальные случаи для индексов $i, j, k$ приводят к комплексно сопряженным тождествам.

Рассмотрим случай, для которого функции $J_{\hat{b}, a}^{c}$ отличны от тождественного нуля. Согласно (5) это означает, что виртуальный тензор отличен от тождественного нуля. Тогда из (13) и (14) получаем

$$
\mu_{2}+\mu_{8}=0, \quad \mu_{4}-\mu_{6}=0, \quad \mu_{1}+\mu_{7}=0, \quad \mu_{3}-\mu_{5}=0 .
$$

Проальтернируем соотношение (15) по индексам $a, b$ :

$$
\left(\mu_{2}-\mu_{8}-\mu_{1}+\mu_{7}+i\left(\mu_{4}+\mu_{6}-\mu_{3}-\mu_{5}\right)\right) J_{[a, b]}^{\hat{c}}=0 .
$$

Просимметризуем соотношение (15) по индексам $a, b$ :

$$
\left(\mu_{2}-\mu_{8}+\mu_{1}-\mu_{7}+i\left(\mu_{4}+\mu_{6}+\mu_{3}+\mu_{5}\right)\right) J_{(a, b)}^{\hat{c}}=0 .
$$

Пусть $J_{[a, b]}^{\hat{c}}$ отличны от тождественного нуля. Тогда согласно (6) структурный тензор отличен от тождественного нуля. В этом случае из (17) получаем

$$
\mu_{2}-\mu_{8}-\mu_{1}+\mu_{7}=0, \quad \mu_{4}+\mu_{6}-\mu_{3}-\mu_{5}=0 .
$$

Пусть $J_{(a, b)}^{\hat{c}}$ отличны от тождественного нуля. Тогда согласно $(6),(10)$ получаем, что $B_{(b c) a}$ отличны от тождественного нуля, т.е. альтернированные компоненты структурного тензора не кососимметричны по первым двум индексам. В этом случае из (18) получаем

$$
\mu_{2}-\mu_{8}+\mu_{1}-\mu_{7}=0, \quad \mu_{4}+\mu_{6}+\mu_{3}+\mu_{5}=0 .
$$

Объединяя (16), (19), (20) получаем, что $\mu_{\beta}=0, \beta=1, \ldots, 8$.

Отметим, что условие $B_{(b c) a}=0$ вместе с условием $B_{a(b c)}=0$ с помощью стандартной процедуры восстановления тождеств записываются в виде $\nabla_{X}(J) X-\nabla_{J X}(J)(J X)=0$. Мы доказали следующую теорему.

Теорема 1. Пространство связностей (11) является 
(1) 8-мерным афбинным пространством тогда и толъко тогда, когда виртуалъный тензор почти эрмитова многообразия отличен от тождественного нуля и

$$
\nabla_{X}(J) X-\nabla_{J X}(J)(J X) \neq 0 ;
$$

(2) 6-мерным аффинным пространством тогда и только тогда, когда виртуальный и структурный тензоры почти эрмитова многообразия отличны от тождественного нуля и

$$
\nabla_{X}(J) X-\nabla_{J X}(J)(J X)=0 ;
$$

(3) 4-мерным афбинным пространством тогда и только тогда, когда виртуальный тензор отличен от тождественного нуля, а структурный тензор - тождественный нуль;

(4) состоит только из римановой связности метрики g тогда и только тогда, когда структурныц и виртуальный тензоры - тождественные нули.

Напомним (см. [2]), что тензорное поле $Q$, определяемое формулой $Q=-\Delta g$, где $\Delta$ - произвольная аффинная связность, $g$ - псевдориманова метрика, называется тензором неметричности. Если

$$
Q(X, Y, Z)=W(X) g(Y, Z),
$$

где $W$-1-форма (она называется формой Вейля), то говорят, что пара $(\Delta, g)$ задает на гладком многообразии геометрию Римана-Картана-Вейля (см. [2]). Если $Q=0$ и $S \neq 0$, $S$-тензор кручения связности $\Delta$, то говорят, что связность $\Delta$ задает геометрию Римана-Картана. Если $Q=W \otimes g$ и $S=0$, то говорят, что связность $\Delta$ задает геометрию Римана-Вейля. Если $Q=0$ и $S=0$, то связность $\Delta$ является римановой. Будем говорить в этом случае, что задана геометрия Римана.

Выясним, какие из связностей (11) задают геометрию Римана-Картана-Вейля.

Вычислим тензор неметричности для связностей (11). По определению тензора неметричности имеем

$$
-Q(X, Y, Z)=\Delta_{X}(g)(Y, Z)=X(g(Y, Z))-g\left(\Delta_{X} Y, Z\right)-g\left(Y, \Delta_{X} Z\right) .
$$

Тогда с учетом (11) и ковариантного постоянства $g$ в связности $\nabla$, получим

$$
\begin{aligned}
& Q(X, Y, Z)=\mu_{2}\left(g\left(\nabla_{Y}(J) X, Z\right)+g\left(\nabla_{Z}(J) X, Y\right)\right)+\mu_{4}\left(g\left(\nabla_{Y}(J)(J X), Z\right)+\right. \\
&\left.g\left(\nabla_{Z}(J)(J X), Y\right)\right)+\mu_{6}\left(g\left(\nabla_{J Y}(J) X, Z\right)+g\left(\nabla_{J Z}(J) X, Y\right)\right)+ \\
&+\mu_{8}\left(g\left(\nabla_{J Y}(J)(J X), Z\right)+g\left(\nabla_{J Z}(J)(J X), Y\right)\right) .
\end{aligned}
$$

На пространстве расслоения $A$-реперов имеем

$$
\begin{aligned}
Q_{i j k}=\mu_{2}\left(g_{l k} J_{i, j}^{l}+g_{l j} J_{i, k}^{l}\right)+\mu_{4} & \left(g_{l k} J_{t, j}^{l} J_{i}^{t}+g_{j l} J_{t, k}^{l} J_{i}^{t}\right)+ \\
& +\mu_{6}\left(g_{l k} J_{i, t}^{l} J_{j}^{t}+g_{l j} J_{i, t}^{l} J_{k}^{t}\right)+\mu_{8}\left(g_{l k} J_{t, m}^{l} J_{i}^{t} J_{j}^{m}+g_{l j} J_{t, m}^{l} J_{i}^{t} J_{k}^{m}\right) .
\end{aligned}
$$

В частности, при $i=a, j=b, k=c$ с учетом (2) получим

$$
Q_{a b c}=\left(\mu_{2}-\mu_{8}+i\left(\mu_{4}+\mu_{6}\right)\right)\left(\varepsilon(c) J_{a, b}^{\hat{c}}+\varepsilon(b) J_{a, c}^{\hat{b}}\right) .
$$

При $i=\hat{a}, j=b, k=c$ получим $Q_{\hat{a} b c}=0$. При $i=a, j=\hat{b}, k=c$ получим

$$
Q_{a \hat{b} c}=\left(\mu_{2}+\mu_{8}+i\left(\mu_{4}-\mu_{6}\right)\right)(-2 i) \varepsilon(a) \varepsilon(c) B_{c a}{ }^{b} .
$$

Остальные группы компонент получаются из вычисленных с помощью операции комплексного сопряжения и свойства симметричности $Q$ по последним двум аргументам.

Условие (21), определяющее геометрию Римана-Картана-Вейля, на пространстве расслоения $A$-реперов с учетом (2) примет вид

$$
Q_{a b c}=0, \quad Q_{\hat{a} b c}=0, \quad Q_{a \hat{b} c}=W_{a} \varepsilon(b) \delta_{c}^{b},
$$


где $\left\{W_{a}, W_{\hat{a}}\right\}$ - компоненты формы Вейля на пространстве расслоения $A$-реперов. Тогда условия того, что $\Delta$ задает геометрию Римана-Картана-Вейля примут вид

$$
\begin{gathered}
\left(\mu_{2}-\mu_{8}+i\left(\mu_{4}+\mu_{6}\right)\right)\left(\varepsilon(c) J_{a, b}^{\hat{c}}+\varepsilon(b) J_{a, c}^{\hat{b}}\right)=0, \\
W_{a} \varepsilon(c) \delta_{c}^{b}=\left(\mu_{2}+\mu_{8}+i\left(\mu_{4}-\mu_{6}\right)\right)(-2 i) \varepsilon(a) \varepsilon(c) B_{c a}{ }^{b} .
\end{gathered}
$$

Умножим обе части второго из полученных равенств на $\varepsilon(c) \varepsilon(a)$, свернем по индексам $a, b$ и учтем (7):

$$
\varepsilon(c) W_{c}=-i(n-1)\left(\mu_{2}+\mu_{8}+i\left(\mu_{4}-\mu_{6}\right)\right) \xi^{\hat{c}} .
$$

Умножим обе части равенства на $\varepsilon(c)$ и учтем $(3)$ :

$$
W_{c}=-i(n-1)\left(\mu_{2}+\mu_{8}+i\left(\mu_{4}-\mu_{6}\right)\right) \alpha_{c} .
$$

Сворачивая второе равенство из $(22)$ и рассуждая аналогично, получим

$$
n W_{a}=i(n-1)\left(\mu_{2}+\mu_{8}+i\left(\mu_{4}-\mu_{6}\right)\right) \alpha_{a} .
$$

Сравнивая $(23)$ и $(24)$, получим $W_{c}=0$. Следовательно, $W=0$ и $Q=0$.

Таким образом, если связность $\Delta$ из (11) задает геометрию Римана-Картана-Вейля, то тензор неметричности должен быть равен нулю, т.е. эта связность задает геометрию Римана-Картана. Такие связности действительно существуют. В самом деле, в случае 8-мерного пространства связностей (11) из первого равенства (22) с учетом леммы 1 получаем

$$
\left(\mu_{2}-\mu_{8}+i\left(\mu_{4}+\mu_{6}\right)\right) \varepsilon(a)\left(J_{c, b}^{\hat{a}}+J_{b, c}^{\hat{a}}\right)=0 .
$$

В случае 8-мерного пространства $J_{(c, b)}^{\hat{a}} \neq 0$, и мы получаем

$$
\mu_{2}-\mu_{8}=0, \quad \mu_{4}+\mu_{6}=0 .
$$

Из второго равенства (22) (при $W_{a}=0$, учитывая, что виртуальный тензор не нулевой) получаем

$$
\mu_{2}+\mu_{8}=0, \quad \mu_{4}-\mu_{6}=0 .
$$

Объединяя полученные уравнения, имеем

$$
\mu_{2}=\mu_{4}=\mu_{6}=\mu_{8}=0 .
$$

Это 4-плоскость в 8-мерном аффинном пространстве.

Вычислим тензор кручения для связностей $\Delta$ из (11). По определению тензора кручения с учетом (11) имеем

$$
\begin{aligned}
S(X, Y)= & \Delta_{X} Y-\Delta_{Y} X-[X, Y]=\left(\mu_{1}-\mu_{2}\right)\left(\nabla_{X}(J) Y-\nabla_{Y}(J) X\right)+ \\
+\left(\mu_{3}-\mu_{6}\right)\left(\nabla_{J X}(J) Y-\nabla_{J Y}(J) X\right)+ & \left(\mu_{4}-\mu_{5}\right)\left(\nabla_{Y}(J)(J X)-\nabla_{X}(J)(J Y)\right)+ \\
& \quad+\left(\mu_{7}-\mu_{8}\right)\left(\nabla_{J X}(J)(J Y)-\nabla_{J Y}(J)(J X)\right) .
\end{aligned}
$$

Для полученной 4-плоскости связностей тензор кручения будет иметь вид

$$
\begin{aligned}
S(X, Y)=\mu_{1}\left(\nabla_{X}(J) Y-\right. & \left.\nabla_{Y}(J) X\right)+\mu_{3}\left(\nabla_{J X}(J) Y-\nabla_{J Y}(J) X\right)- \\
& -\mu_{5}\left(\nabla_{Y}(J)(J X)-\nabla_{X}(J)(J Y)\right)+\mu_{7}\left(\nabla_{J X}(J)(J Y)-\nabla_{J Y}(J)(J X)\right) .
\end{aligned}
$$

В силу теоремы 1 тензор кручения будет тождественным нулем тогда и только тогда, когда $\mu_{1}=\mu_{3}=\mu_{5}=\mu_{7}=0$. Итак, мы доказали следующую теорему.

Теорема 2. В 8-мерном афбинном пространстве связностей (11) множество связностей, задающих геометрию Римана-Картана и геометрию Римана, образуют 4-плоскость.

Рассмотрим тензор кручения (26). Запишем его компоненты на пространстве расслоения $A$ реперов:

$$
\begin{aligned}
S_{i j}{ }^{k}=\left(\mu_{1}-\mu_{2}\right)\left(J_{j, i}^{k}-J_{i, j}^{k}\right)+\left(\mu_{3}-\right. & \left.\mu_{6}\right)\left(J_{j, l}^{k} J_{i}^{l}-J_{i, l}^{k} J_{j}^{l}\right)+ \\
& +\left(\mu_{4}-\mu_{5}\right)\left(J_{l, j}^{k} J_{i}^{l}-J_{l, i}^{k} J_{j}^{l}\right)+\left(\mu_{7}-\mu_{8}\right)\left(J_{l, t}^{k} J_{j}^{l} J_{i}^{t}-J_{l, t}^{k} J_{i}^{l} J_{j}^{t}\right) .
\end{aligned}
$$


В частности, для $i=a, j=b, k=c$ получим $S_{a b}^{c}=0$. Для $i=\hat{a}, j=b, k=c$ получим

$$
S_{\hat{a} b}^{c}=\left(\mu_{2}-\mu_{1}+\mu_{8}-\mu_{7}+i\left(\mu_{6}-\mu_{3}-\mu_{4}+\mu_{5}\right)\right) J_{\hat{a}, b}^{c} .
$$

Для $i=a, j=b, k=\hat{c}$ получим

$$
S_{a b}^{\hat{c}}=\left(\mu_{2}-\mu_{1}+\mu_{7}-\mu_{8}+i\left(\mu_{6}-\mu_{3}+\mu_{4}-\mu_{5}\right)\right)\left(J_{a, b}^{\hat{c}}-J_{b, a}^{\hat{c}}\right) .
$$

Остальные компоненты тензора кручения получаются с помощью комплексного сопряжения и в силу кососимметричности $S$.

Отметим, что среди связностей (11) нет связностей, которые задают геометрию Римана-Вейля, так как условие $Q=W \otimes g$ выполняется только в случае $Q=0$. Но если оставить только требование $S=0$, то получим (в случае 8-мерного пространства связностей (11)) 4-плоскость связностей, удовлетворяющих этому условию:

$$
\mu_{1}-\mu_{2}=\mu_{3}-\mu_{6}=\mu_{4}-\mu_{5}=\mu_{7}-\mu_{8}=0 .
$$

Получаем, что в случае 8-мерного пространства связности (11), удовлетворяющие условию $Q=0$, и связности (11), удовлетворяющие условию $S=0$, образуют 4-плоскости, имеющие единственную общую точку - риманову связность.

4. Тензор кручения. Рассмотрим подробнее тензор кручения в случае 8-мерного пространства связностей. На пространстве расслоения $A$-реперов он имеет две существенные ненулевые группы компонент: $\left\{S_{\hat{a} b}^{c}\right\}$ и $\left\{S_{a b}{ }^{\hat{c}}\right\}$. Выясним геометрический смысл обращения в нуль каждой из указанных групп компонент.

Пусть $S_{\hat{a} b}^{c}=0$. Множество таких связностей определяется уравнениями

$$
\mu_{2}-\mu_{1}+\mu_{8}-\mu_{7}=0, \quad \mu_{6}-\mu_{3}-\mu_{4}+\mu_{5}=0 .
$$

Следовательно, такие связности образуют 6-плоскость в 8-мерном пространстве. Применим стандартную процедуру восстановления тождеств для $S_{\hat{a} b}^{c}=S_{a \hat{b}}^{c}=S_{a b}^{c}=0$. Имеем $S_{i b}{ }^{c}=0$ и $S_{a j}{ }^{c}=0$. Эти равенства равносильны

$$
\sigma S^{\mathbb{C}}(X, \sigma Y)=0, \quad \sigma S^{\mathbb{C}}(\sigma X, Y)=0 .
$$

Применяя определение проекторов $(1)$ и $\mathbb{C}$-линейность комплексификации $S^{\mathbb{C}}$ тензора кручения $S$, получим

$$
J S(X, Y)+S(X, J Y)=0, \quad J S(X, Y)+S(J X, Y)=0 .
$$

Применим стандартную процедуру восстановления тождеств к группе компонент $\left\{S_{a b} \hat{c}\right\}$. С учетом (27) получим

Тогда

$$
S_{a b}{ }^{\hat{c}}=2\left(\left(\mu_{2}-\mu_{1}\right)+i\left(\mu_{4}-\mu_{5}\right)\right)\left(J_{a, b}^{\hat{c}}-J_{b, a}^{\hat{c}}\right) .
$$

$$
\bar{\sigma} S^{\mathbb{C}}(\sigma X, \sigma Y)=2\left(\left(\mu_{2}-\mu_{1}\right)+i\left(\mu_{4}-\mu_{5}\right)\right)\left(\bar{\sigma} \nabla_{\sigma Y}\left(J^{\mathbb{C}}\right)(\sigma X)-\bar{\sigma} \nabla_{\sigma X}\left(J^{\mathbb{C}}\right) \sigma Y\right) .
$$

С учетом определения проекторов (1) и соотношений (28) получим

$$
\begin{aligned}
S(X, Y)+i J S(X, Y) & =\left(\left(\mu_{2}-\mu_{1}\right)+i\left(\mu_{4}-\mu_{5}\right)\right)\left\{\nabla_{Y}(J) X-\nabla_{X}(J) Y+\nabla_{J X}(J)(J Y)-\right. \\
& \left.-\nabla_{J Y}(J)(J X)+i\left(\nabla_{J X}(J) Y+\nabla_{X}(J)(J Y)-\nabla_{J Y}(J) X-\nabla_{Y}(J)(J X)\right)\right\} .
\end{aligned}
$$

Приравнивая вещественную и мнимую части, получаем два равенства. Легко видеть, что второе равенство получается из первого, если на него подействовать $J$ и применить тождество $J \circ \nabla_{X}(J)=-\nabla_{X}(J) \circ J$. Поэтому выпишем только первое равенство:

$$
\begin{aligned}
S(X, Y)=\left(\mu_{2}-\mu_{1}\right)\left(\nabla_{Y}(J) X\right. & \left.-\nabla_{X}(J) Y+\nabla_{J X}(J)(J Y)-\nabla_{J Y}(J)(J X)\right)- \\
& -\left(\mu_{4}-\mu_{5}\right)\left(\nabla_{J X}(J) Y+\nabla_{X}(J)(J Y)-\nabla_{J Y}(J) X-\nabla_{Y}(J)(J X)\right) .
\end{aligned}
$$

Сравнивая это равенство с (4), получаем

$$
S(X, Y)=4\left(\mu_{2}-\mu_{1}\right)(C(X, J Y)-C(Y, J X))-4\left(\mu_{4}-\mu_{5}\right)(C(X, Y)-C(Y, X)) .
$$

Итак, мы получаем следующее утверждение. 
Теорема 3. В 8-мерном пространстве связностей (11) 6-плоскость связностей имеет тензор кручения, определяемый только структурным тензором.

Хорошо известно, что при конформных преобразованиях почти эрмитовой структуры (т.е. при переходе от структуры $(J, g)$ к $\left(J, e^{2 f} g\right), f$ - гладкая функция на многообразии $\left.M\right)$ структурный тензор является инвариантным.

Следствие 1. В 8-мерном пространстве связностей (11) 6-плоскость связностей имеет тензор кручения, инвариантный относительно конформных преобразований почти эрмитовой структуры.

Напомним, что тензором Нейенхейса почти комплексной структуры $J$ (см. [4, с. 270]) называется тензорное поле типа $(2,1)$, определяемое формулой

$$
N(X, Y)=\frac{1}{4}(-[X, Y]+[J X, J Y]-J[J X, Y]-J[X, J Y]) .
$$

Если кроме почти комплексной структуры $J$ на многообразии задана метрика $g$, согласованная с $J$, то для римановой связности $\nabla$ получаем

$$
N(X, Y)=\frac{1}{4}\left(\nabla_{J X}(J) Y+\nabla_{X}(J)(J Y)-\nabla_{J Y}(J) X-\nabla_{Y}(J)(J X)\right)=C(X, Y)-C(Y, X) .
$$

Если к условиям (27), задающим связности с кручением вида (29) добавить требование $\mu_{2}-\mu_{1}=$ 0 , то получим 5-плоскость связностей, у которых тензор кручения с точностью до константы совпадает с тензором Нейенхейса. Если к этому добавить еще и требование $-4\left(\mu_{4}-\mu_{5}\right)=1$, то получаем 4-плоскость связностей с тензором кручения, равным тензору Нейенхейса.

Следствие 2. В 8-мерном пространстве связностей (11) 5-плоскость связностей имеет тензор кручения, совпадающий с точностью до константы с тензором Нейенхейса. При этом для 4-плоскости связностей тензор кручения совпадает с тензором Нейенхейса.

Пусть теперь $S_{a b}{ }^{\hat{c}}=0$. Множество таких связностей задается уравнениями

$$
\mu_{2}-\mu_{1}+\mu_{7}-\mu_{8}=0, \quad \mu_{6}-\mu_{3}+\mu_{4}-\mu_{5}=0 .
$$

Они задают 6-плоскость в 8-мерном пространстве. Для компонент тензора кручения таких связностей имеем $S_{a b}{ }^{k}=0$. Применяя стандартную процедуру восстановления тождеств, получим

$$
S(X, Y)=S(J X, J Y), \quad S(J X, Y)=-S(X, J Y) .
$$

Второе равенство получается из первого при замене $X$ на $J X$.

Кроме того, с учетом (30) для оставшихся ненулевых компонент тензора кручения получаем

$$
S_{\hat{a} b}^{c}=2\left(\mu_{2}-\mu_{1}+i\left(\mu_{5}-\mu_{4}\right)\right) J_{\hat{a}, b}^{c} .
$$

В силу кососимметричности тензора кручения из этого равенства получаем

$$
S_{a \hat{b}}^{c}=-2\left(\mu_{2}-\mu_{1}+i\left(\mu_{5}-\mu_{4}\right)\right) J_{\hat{b}, a}^{c} .
$$

Применяя к каждому из двух последних равенств стандартную процедуру восстановления тождеств, получим

$$
\begin{aligned}
S(X, Y)+J S(J X, Y)=2\left(\mu_{2}-\mu_{1}\right)\left(\nabla_{Y}(J) X\right. & \left.+\nabla_{J Y}(J) J X\right)- \\
& -2\left(\mu_{5}-\mu_{4}\right)\left(-\nabla_{J Y}(J) X+\nabla_{Y}(J) J X\right), \\
S(X, Y)-J S(J X, Y)=-2\left(\mu_{2}-\mu_{1}\right)\left(\nabla_{X}(J) Y\right. & \left.+\nabla_{J X}(J) J Y\right)+ \\
& +2\left(\mu_{5}-\mu_{4}\right)\left(-\nabla_{J X}(J) Y+\nabla_{X}(J) J Y\right) .
\end{aligned}
$$

Складывая два последних равенства и учитывая (4), получим

$$
S(X, Y)=4\left(\mu_{2}-\mu_{1}\right)(B(X, J Y)-B(Y, J X))-4\left(\mu_{5}-\mu_{4}\right)(B(X, Y)-B(Y, X)) .
$$

Итак, получаем следующую теорему. 
Теорема 4. В 8-мерном пространстве связностей (11) 6-плоскость связностей имеет тензор кручения, определяемый только виртуальным тензором.

Если добавить к условиям (30) еще требование $\mu_{2}-\mu_{1}=0$, то получим следующее утверждение.

Следствие 3. В 8-мерном пространстве связностей (11) 5-плоскость связностей имеет тензор кручения, совпадающий с точностью до константы с альтерначией виртуального тензора. В частности, для 4-плоскости связностей тензор кручения совпадает с альтернацией виртуального тензора.

5. Ковариантный дифференциал $J$. Вычислим ковариантный дифференциал $J$ для связностей (11). Имеем

$$
\begin{aligned}
& \Delta_{X}(J) Y=\Delta_{X}(J Y)-J \Delta_{X} Y=\nabla_{X}(J) Y+2 \mu_{1} \nabla_{X}(J)(J Y)+ \\
& +\left(\mu_{2}-\mu_{8}\right)\left(\nabla_{Y}(J)(J X)+\nabla_{J Y}(J) X\right)+2 \mu_{3} \nabla_{J X}(J)(J Y)+ \\
& \quad+\left(\mu_{4}+\mu_{6}\right)\left(\nabla_{J Y}(J)(J X)-\nabla_{Y}(J) X\right)-2 \mu_{5} \nabla_{X}(J) Y-2 \mu_{7} \nabla_{J X}(J) Y .
\end{aligned}
$$

На пространстве расслоения $A$-реперов имеем

$$
\begin{aligned}
J_{j \mid i}^{k}=J_{j, i}^{k}+2 \mu_{1} J_{l, i}^{k} J_{j}^{l}+\left(\mu_{2}-\mu_{8}\right)\left(J_{l, j}^{k} J_{i}^{l}+J_{i, l}^{k} J_{j}^{l}\right) & +2 \mu_{3} J_{l, t}^{k} J_{j}^{l} J_{i}^{t}+ \\
& +\left(\mu_{4}+\mu_{6}\right)\left(J_{l, t}^{k} J_{i}^{l} J_{j}^{t}-J_{i, j}^{k}\right)-2 \mu_{5} J_{j, i}^{k}-2 \mu_{7} J_{j, l}^{k} J_{i}^{l} .
\end{aligned}
$$

В частности, при $i=a(i=\hat{a}), j=b, k=c$ получаем

$$
J_{b \mid a}^{c}=J_{b, a}^{c}=0 \quad\left(J_{b \mid \hat{a}}^{c}=J_{b, \hat{a}}^{c}=0\right) .
$$

При $i=a, j=\hat{b}, k=c$ получаем

$$
J_{\hat{b} \mid a}^{c}=\left(1+2 \mu_{3}-2 \mu_{5}-2 i\left(\mu_{1}+\mu_{7}\right)\right) J_{\hat{b}, a}^{c} .
$$

При $i=a, j=b, k=\hat{c}$ получаем

$$
J_{b \mid a}^{\hat{c}}=\left(1-2 \mu_{3}-2 \mu_{5}+2 i\left(\mu_{1}-\mu_{7}\right)\right) J_{b, a}^{\hat{c}}+\left(2 i\left(\mu_{2}-\mu_{8}\right)-2\left(\mu_{4}+\mu_{6}\right)\right) J_{a, b}^{\hat{c}} .
$$

Хорошо известно, что связность $\Delta$ является почти комплексной тогда и только тогда, когда $\Delta J=0$. Тогда с учетом (33) и (34) в 8-мерном пространстве множество почти комплексных связностей задается уравнениями

$$
1+2 \mu_{3}-2 \mu_{5}=0, \quad \mu_{1}+\mu_{7}=0, \quad 1-2 \mu_{3}-2 \mu_{5}=0, \quad \mu_{1}-\mu_{7}=0, \quad \mu_{2}-\mu_{8}=0, \quad \mu_{4}+\mu_{6}=0 .
$$

Решая эту систему, получаем, что множество почти комплексных связностей (11) образует 2плоскость, задаваемую уравнениями

$$
\mu_{1}=\mu_{3}=\mu_{7}=0, \quad \mu_{2}-\mu_{8}=0, \quad \mu_{4}+\mu_{6}=0, \quad \mu_{5}=\frac{1}{2} .
$$

Напомним, что связность $\Delta$ является почти эрмитовой тогда и только тогда, когда $\Delta J=\Delta g=0$. Тогда к полученным уравнениям добавляем (25), задающие $\Delta g=0$, и получаем единственную почти эрмитову связность. Тем самым доказана следующая теорема.

Теорема 5. В 8-мерном пространстве связностей (11) почти комплексные связности образуют 2-плоскость. Существует единственная почти эрмитова связность

$$
\Delta_{X} Y=\nabla_{X} Y+\frac{1}{2} \nabla_{X}(J)(J Y)
$$

Отметим, что кручение полученной почти эрмитовой связности

$$
S(X, Y)=\nabla_{X}(J)(J Y)-\nabla_{Y}(J)(J X)
$$

отлично от тензора Нейенхейса. 
Выясним, существуют ли среди связностей (11) такие, для которых ковариантный дифференциал $J$ определяется только структурным тензором почти эрмитовой структуры $(J, g)$. Из равенств (33), (34) следует, что искомые связности задаются уравнениями

$$
1+2 \mu_{3}-2 \mu_{5}=0, \quad \mu_{1}+\mu_{7}=0, \quad 1-2 \mu_{3}-2 \mu_{5}=2\left(\mu_{4}+\mu_{6}\right), \quad \mu_{1}-\mu_{7}=-\left(\mu_{2}-\mu_{8}\right) .
$$

Эти уравнения задают 4-плоскость в 8-мерном пространстве связностей. Так как для рассматриваемых связностей выполняется $J_{\hat{b} \mid a}^{c}=0$, то с учетом (32) получим $J_{j \mid a}^{c}=0$. Применяя к этим соотношениям стандартную процедуру восстановления тождеств, получим

$$
\Delta_{X}(J) Y-J \Delta_{J X}(J) Y=0 .
$$

Найдем явный вид для $\Delta_{X}(J) Y$, используя стандартную процедуру восстановления тождеств для (34). Тогда с учетом (36) получим:

$$
\Delta_{X}(J) Y=2\left(1-2 \mu_{3}-2 \mu_{5}\right)(C(Y, J X)-C(X, J Y))+4\left(\mu_{1}-\mu_{7}\right)(C(X, Y)-C(Y, X)) .
$$

Учитывая (35), получим

$$
\Delta_{X}(J) Y=4\left(1-2 \mu_{5}\right)(C(Y, J X)-C(X, J Y))+8 \mu_{1}(C(X, Y)-C(Y, X)) .
$$

Таким образом, мы доказали следующую теорему.

Теорема 6. В 8-мерном пространстве связностей (11) 4-плоскость связностей имеет ковариантный дифберенциал $J$, определяемый только структурным тензором.

Следствие 4. Для связностей из теоремы 6 ковариантный дифференциал $J$ инвариантен при конформных преобразованиях почти эрмитовой структуры.

Если к условиям (35) добавить требование $1-2 \mu_{3}-2 \mu_{5}=0$, то получим 3 -плоскость связностей, для которых $\Delta J$ с точностью до константы совпадает с тензором Нейенхейса почти комплексной структуры $J$. Если еще добавить условие $4\left(\mu_{1}-\mu_{7}\right)=1$, то получим 2 -плоскость связностей, для которых $\Delta J$ равен тензору Нейенхейса.

Найдем связности, для которых тензор кручения и $\Delta J$ будут определяться только структурным тензором (а значит, будут инвариантны при конформных преобразованиях почти эрмитовой структуры). Объединяя условия (35) и (27) получим уравнения

$$
\mu_{1}=-\mu_{7}, \quad \mu_{2}=\mu_{7}, \quad \mu_{4}=\frac{1}{4}-\mu_{3}, \quad \mu_{5}=\frac{1}{2}+\mu_{3}, \quad \mu_{6}=-\frac{1}{4}-\mu_{3}, \quad \mu_{8}=-\mu_{7} .
$$

Они задают в 8-мерном пространстве 2-плоскость связностей.

Наконец, найдем связности, для которых $\Delta J$ определяются только виртуальным тензором. Из (34) получим

$$
1-2 \mu_{3}-2 \mu_{5}=0, \quad \mu_{1}-\mu_{7}=0, \quad \mu_{2}-\mu_{8}=0, \quad \mu_{4}+\mu_{6}=0 .
$$

Следовательно, это 4-плоскость в 8-мерном пространстве. Для таких связностей имеем $J_{j \mid a}^{\hat{c}}=0$. Применяя стандартную процедуру восстановления тождеств, получим из этих соотношений

$$
\Delta_{X}(J) Y+J \Delta_{J X}(J) Y=0 .
$$

Получим явный вид рассматриваемых связностей. Применяя стандартную процедуру восстановления тождеств к (33), получаем

$$
\Delta_{X}(J) Y=2\left(1+2 \mu_{3}-2 \mu_{5}\right) B(Y, J X)-2\left(\mu_{1}+\mu_{7}\right) B(Y, X) .
$$

С учетом (37) получаем

$$
\Delta_{X}(J) Y=4\left(1-2 \mu_{5}\right) B(Y, J X)+8 \mu_{1} B(Y, X) .
$$




\section{СПИСОК ЛИТЕРАТУРЫ}

1. Бабурова О. В., Фролов Б. Н. Математические основы современной теории гравитации. - М.: Прометей, 2012.

2. Катанаев М. О. Геометрические методы в математической физике/ arXiv: $1311.0733 v 3$ [math.ph] .

3. Кириченко В. Ф. Обобщенные классы Грея-Хервеллы и голоморфно-проективные преобразования обобщенных почти эрмитовых структур// Изв. РАН. Сер. мат. - 2005. - 69, № 5. - С. 107--132.

4. Кириченко В. Ф. Дифференциально-геометрические структуры на многообразиях. - Одесса: Печатный дом, 2013.

5. Gray A., Hervella L. The sixteen classes of almost Hermitian manifolds and their linear invariants// Ann. Mat. Pura Appl. IV. — 1980. — 123. - P. 35-58.

6. Puetzfeld D., Tresguerres R. A cosmological model in Weyl-Cartan spacetime// Class. Quantum Grav. 2001. - 18. - P. 677-694.

Игнаточкина Лия Анатольевна

Московский педагогический государственный университет

E-mail: ignlia@gmail.com

Горгинян Юлия Ашотовна

Московский педагогический государственный университет

E-mail: kiyulia0@gmail.com 